\title{
Aggregation of blood components on a surface in a microfluidic environment
}

\author{
F.-K. Tsai, J. L. Lauer, and J. L. Shohet ${ }^{a)}$ \\ Plasma Processing and Technology Laboratory, and Department of Electrical and Computer Engineering, \\ University of Wisconsin-Madison, Madison, Wisconsin 53706
}

(Received 25 August 2005; accepted 12 December 2005; published online 25 January 2006)

The aggregation of blood components on the blood-contacting surface of a medical device will reduce its reliability and lifetime. Such aggregations are known to be generated by sheared-flow activation of blood components which themselves are greatly influenced by flow patterns. This is especially important in the case of a microfluidic system. A numerical simulation was conducted to evaluate the flow parameters in a microminiature blood circulation loop to determine those flow factors that promote the aggregation of blood components and their potential deposition on the blood-contacting surface. The local geometry of the system was found to be the most important factor that affects the evolution of the flow field. Based on these results, the predicted locations of aggregation of blood components for the circulating blood-loop system were compared with experimental results. (C) 2006 American Institute of Physics. [DOI: 10.1063/1.2163993]

\section{INTRODUCTION}

Aggregation of blood components, especially platelets, on a blood-contacting surface is an important issue for the design and operation of small-diameter devices through which blood flows, e.g., artificial blood vessels, blood-testing devices, etc. Such aggregations will lead to the formation of clots and will degrade or block the functionalities of the device. Since the use of microfluidic components has become important in developing an equipment for blood analysis, it is critical, during the design process, to be able to predict the locations of possible formation and potential deposition of blood aggregates and to minimize these conditions. Adhesion of blood components to the device walls is known to be closely related to activation of blood components, ${ }^{1}$ such that the adhesion is greatly enhanced by such activation. ${ }^{2}$ It is well known that the activation of platelets in a sheared-flow field is not only dependent on the intensity of shear stress, but also on the time of exposure to the shear-stress field. ${ }^{1}$ Thus, a threshold condition for the activation of platelets can be obtained which is a function of the shear stress and the exposure time of the blood to the shear stress. Calculation of this condition is of great help in the design of medical devices.

A previous study ${ }^{3}$ of the mechanisms of shear-induced platelet activation is shown in Fig. 1 as a locus plot for the shear stress required for platelet activation. The amount of stimulation $^{4}$ (level of activation), ${ }^{5}$ which is proportional to the product of local shear stress and exposure time to that stress, is widely accepted as the index that determines platelet activation in shear flows. The loci for incipient shearrelated platelet serotonin release and red-blood-cell hemolysis are displayed in the figure on a shear-stress-exposuretime phase plane plot. For conditions above the loci, the platelets and/or the red blood cells are activated. The threshold stresses for activation were found by extrapolation of the

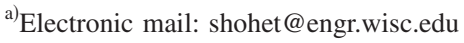

results from several experiments (represented by different types of symbols on the figure) so that the curves in Fig. 1 can be used to find the platelet activation threshold.

We will assume that blood is a Newtonian fluid in our model since past numerical studies concluded that there were only small differences in shear stress between the results by treating blood as a non-Newtonian rather than a Newtonian fluid. Accordingly, the blood was assumed to be an incom-

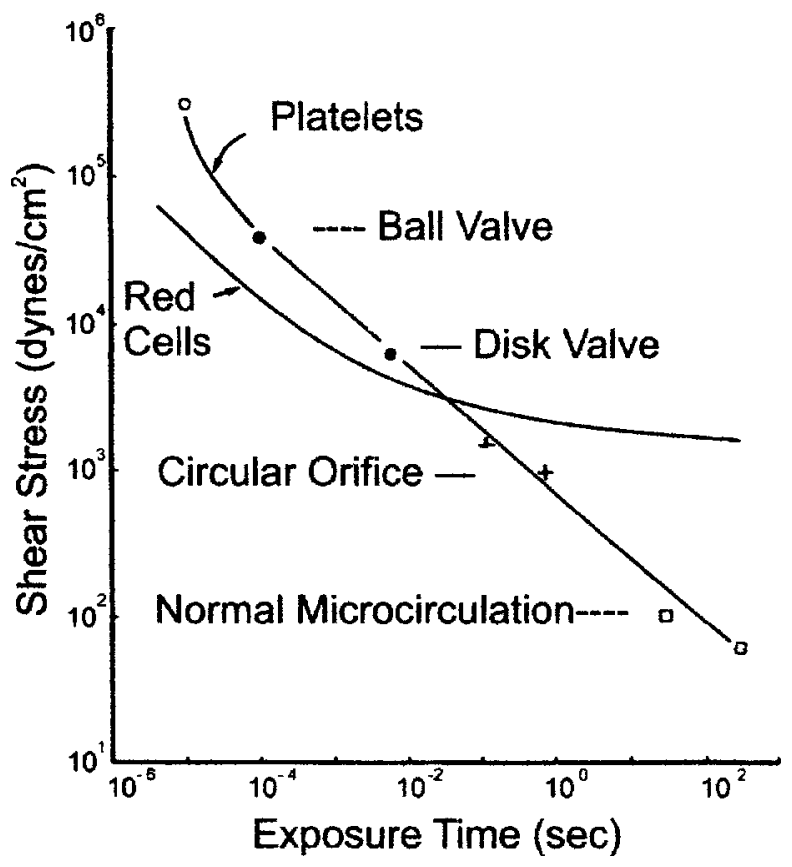

FIG. 1. The locus of incipient shear-related serotonin release (plàtelets) and hemolysis (red cells). The experimental results for the serotonin release from platelets by different researchers are marked by different types of symbol. The locus for threshold stress is based on the extrapolation of those results. Estimates of shear-stress levels for different medical conditions from cardiac valves to the normal microcirculation are also indicated by short horizontal lines in the figure. For example, the estimated shear-stress level in microcirculation is in the order of $10^{2}$ dynes $/ \mathrm{cm}^{2}$, whereas the shear stress level in cardiac valves is above $10^{4} \mathrm{dyn} / \mathrm{cm}^{2}$ (adapted from Ref. 3). 
pressible Newtonian fluid with a density of $1050 \mathrm{~kg} \mathrm{~m}^{-3}$ and a viscosity of $3.5 \times 10^{-3} \mathrm{~kg} \mathrm{~m}^{-1} \mathrm{~s}^{-1}$. 5 .6 For simple shear flow or laminar flow of a Newtonian fluid in a tube, the shear stress reduces to the velocity gradient times the viscosity. The wall shear stress for a Newtonian fluid is thus given as

$$
\tau_{w}=-\mu\left(\frac{\partial u_{\text {axial }}}{\partial r}\right)_{r=R},
$$

where $u_{\text {axial }}$ is the axial velocity, $r$ is the distance in the radial direction from the center of the tubing, $R$ is the tube radius, and $\mu$ is the blood shear viscosity. We also define the shear rate $\dot{\gamma}$ as

$$
\dot{\gamma}=-\frac{\partial u_{\mathrm{axial}}}{\partial r}
$$

for each value of $r$.

Previous works ${ }^{5,7-11}$ showed that the location of aggregation of blood components could be determined from variations in the flow field, i.e., acceleration, deceleration, and nonuniform patterns. In particular, the shear stress in the flow field can lead to the deformation and/or rolling of the blood components on the endothelial surface and/or a device wall. ${ }^{2,12}$ Blood components that experience sufficiently high levels of shear stress will be activated and are likely to adhere to each other and are then likely to form adherent structures on the blood-material interface. These aggregations, if trapped in recirculation zones (vortices), will collect even more components that pass by in the blood. ${ }^{7}$ Some of the aggregates that originate in vortex-shedding regions, which are unsteady flows generated when a fluid is flowing over a blunt object, can form free emboli, which will be transported downstream from their birthplace, increasing the risk of widespread deposition of blood components along the entire length of the system. Another important factor that could affect the growth of aggregates in the recirculation zone is the circulation rate of the flow or vorticity, $\boldsymbol{\Omega}=\boldsymbol{\nabla} \times \mathbf{V}$, where $\mathbf{V}$ is the flow velocity. $\boldsymbol{\Omega}$ is nonzero almost everywhere outside the recirculation zone in shear flow since it will bring together the aggregated platelets in a closed environment for more frequent and prolonged collisions with each other. Previous studies also concluded that rolling of blood components (e.g., leukocytes) serves to recruit additional leukocytes. ${ }^{13,14}$ Both these mechanisms can largely increase the size of the aggregate and thus could result in a significantly larger density of deposited blood components. ${ }^{10}$

To proceed, a model system, which we represent as microfluidic blood-circulation loops connected together with tubings of different sizes, was examined experimentally for deposition of blood aggregates and then simulated with a computational fluid dynamics (CFD) code. This system was selected because it has local changes in geometry along its length that are very common in typical microfluidic devices. To develop a predictive model, the CFD simulation was conducted to calculate the blood-flow parameters in the microfluidic recirculating loop system. A numerical threshold criterion $^{4,5}$ for platelet aggregation that depends on flow parameters will be introduced.

\section{MODEL SYSTEM}

\section{A. Experiment}

Heparinized human blood was passed through a microfluidic circulation loop driven by a peristaltic pump (as shown in Fig. 2) and subsequently examined for aggregates of blood components. The polyethylene (PE) tubing inside the peristaltic pump (large tubing) had an inner diameter of $2.1 \mathrm{~mm}$ and a length of $14 \mathrm{~cm}$. Both sections of the PE tubing outside the peristaltic pump (small tubing) had an inner diameter of $1.14 \mathrm{~mm}$ and a length of $26 \mathrm{~cm}$. The direction of blood flow is shown in Fig. 2. Both the flow pattern and the blood adhesion were examined in six separate regions (A-F) which are indicated in the figure. We identify them along with locations in the model system $(\mathrm{G}-\mathrm{L})$ where the adhesion of blood components was measured (see Table I).

Twenty milliliters of blood was obtained from a healthy human donor. It was heparinized and stored at $37^{\circ} \mathrm{C}$ for 20 min until it was circulated in the loop for $1 \mathrm{~h}$ with a constant average blood-flow rate of $45 \mathrm{ml} / \mathrm{h}$. This is equivalent to an average velocity in the entrance region of $0.012 \mathrm{~m} / \mathrm{s}$. Following blood exposure, the 26-cm-long PE tubes were cut into $4 \mathrm{~cm}$ sections after fixation and dehydration of the lumenal surface. The fixation was done by first rinsing the sections with phosphate buffer solution (PBS) that was circulated through each section for $5 \mathrm{~min}$. Then, a mixed solution of glutaraldehyde and tannic acid in PBS was flushed through the PE tubes for $15 \mathrm{~min}$. The purpose of the

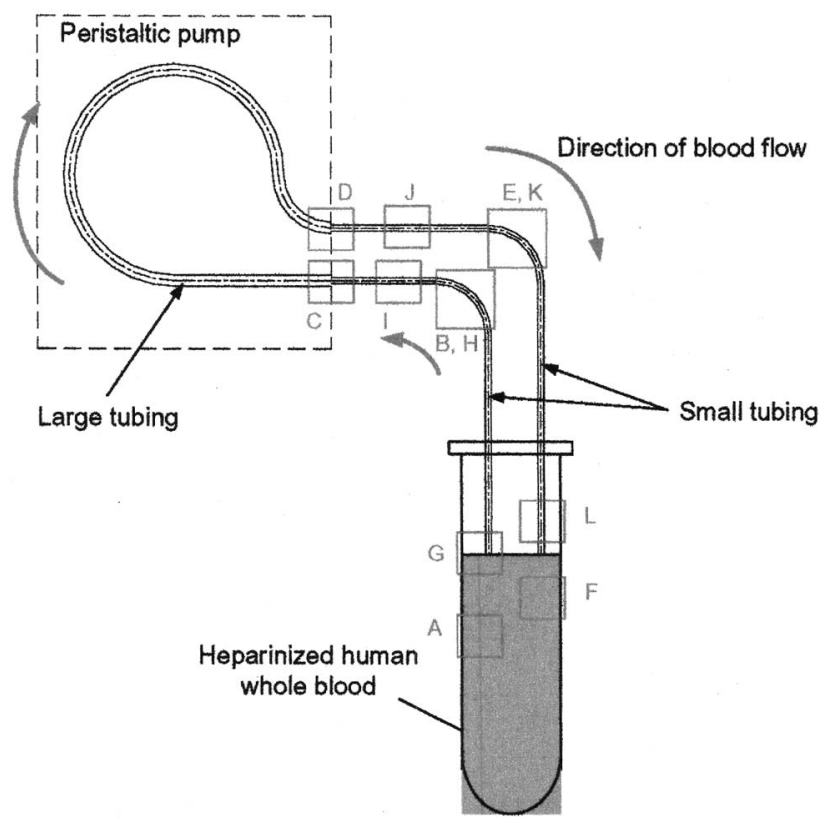

FIG. 2. The PE tubing recirculation loop system used for both the experimental measurements and the numerical solutions for the flow parameters. The tube located inside the peristaltic pump (internal tubing) has a larger inner diameter of $2.1 \mathrm{~mm}$ and a length of $14 \mathrm{~cm}$. The smaller-diameter tubing (external tubing) has an inner diameter of $1.14 \mathrm{~mm}$ and a length of $26 \mathrm{~cm}$ (adapted from Ref. 15). The alphabetized regions will be referred to in subsequent figures. 
TABLE I. Specific locations in the microfluidic circulation loop.

\begin{tabular}{ll}
\hline \hline Label & \multicolumn{1}{c}{ Name } \\
\hline $\mathrm{A}$ & Entrance region \\
$\mathrm{B}$ & Elbow region in inflowing branch \\
$\mathrm{C}$ & Transition region between tubings of different \\
& diameters in inflowing branch \\
$\mathrm{D}$ & Transition region in outflowing branch \\
$\mathrm{E}$ & Elbow region in outflowing branch \\
$\mathrm{F}$ & Exit region \\
$\mathrm{G}$ through L & Locations of blood adhesion measurements \\
\hline \hline
\end{tabular}

glutaraldehyde solution is to fix the platelets and cells that may be attached to the lumenal surface of the tubes, while the tannic acid improves scanning electron microscopy (SEM) imaging. After the fixation step, the sections were again rinsed with PBS. The fixed cells and platelets were dehydrated by flushing the tubes with increasing concentrations of ethanol for 2 min at each incremental step of concentration. The above processes were all done at room temperature using the same flow rate as the blood flow. The glutaraldehyde fixation protocol used for SEM analysis helped the cross-linking of attached platelets, and thus the platelet aggregates would not be washed away in the following rinse steps. The locations of the six sections taken from the upstream and downstream (inflowing and outflowing re- gions) are shown as regions $\mathrm{G}$ through $\mathrm{L}$ in Fig. 2. For SEM imaging, $1 \mathrm{~cm}$ sections were cut from the middle of each of these $4 \mathrm{~cm}$ sections.

Figure 3 shows the SEM images for all six of the $1 \mathrm{~cm}$ sections. It can be seen that the density of platelets adhering to the lumenal surface decreased with distance from the entrance of the inflowing tubing toward the pump and then increased in the outflowing tubing away from the pump until past the "elbow" region shown in Fig. 2. To see this effect more clearly, Fig. 4 shows a graph of the average density of adherents versus distance from the peristaltic pump for both the inflowing and outflowing tubings.

\section{B. Simulation model for recirculating loop system}

The PE tubing recirculation loop model system ${ }^{15}$ shown in Fig. 2 was used as a basis for developing the simulation model. The Navier-Stokes (NS) and continuity (massconservation) equations, including the effects of nonuniform flow, were solved numerically. It was assumed for simplicity that the system produced a steady flow into the entrance region of the microtubing. Since there are geometrical mismatches at the junctions between the tubing and the reservoir, a secondary-flow pattern (recirculation zone) is likely to develop in these regions, which the simulation does not generate.

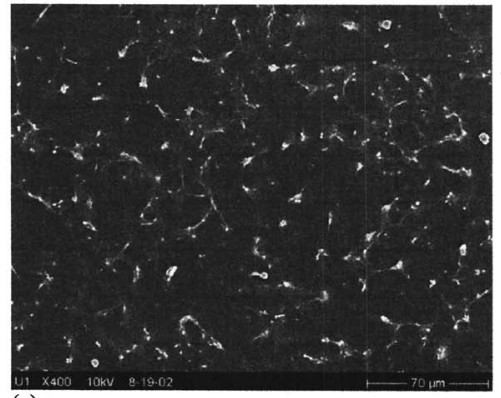

(a)

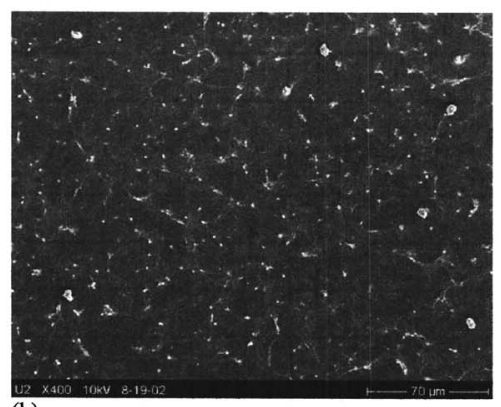

(b)

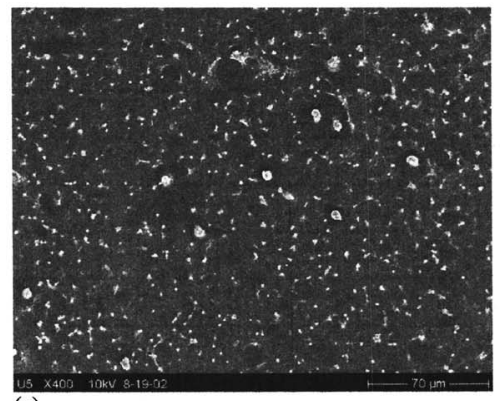

(c)

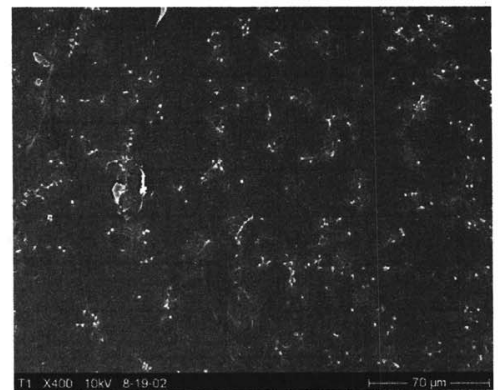

(d)

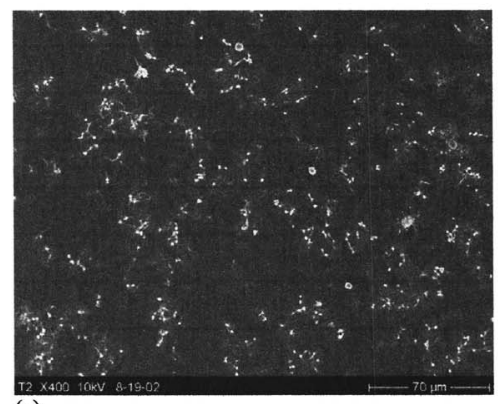

(e)

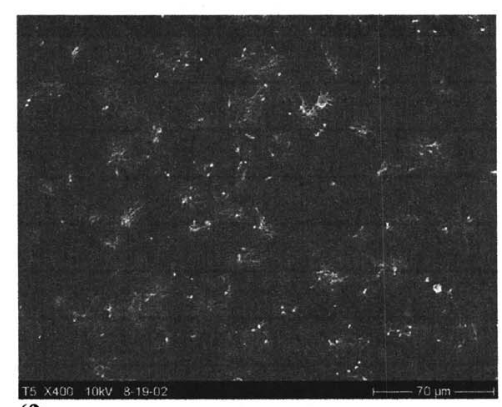

(f)
FIG. 3. SEM images of the platelet deposition on different sections of the external tubings. (a) $1.5-2.5 \mathrm{~cm}$ from the peristaltic pump in the inflowing branch (region I). (b) $5.5-6.5 \mathrm{~cm}$ from the peristaltic pump in the inflowing branch (region $\mathrm{H}$ ). (c) $17.5-18.5 \mathrm{~cm}$ from the peristaltic pump in the inflowing branch (region $\mathrm{G}$ ). (d) $1.5-2.5 \mathrm{~cm}$ from the peristaltic pump in the outflowing branch (region $\mathrm{J}$ ). (e) $5.5-6.5 \mathrm{~cm}$ from the peristaltic pump in the outflowing branch (region K). (f) $17.5-18.5 \mathrm{~cm}$ from the peristaltic pump in the outflowing branch (region L). The results show a higher density of platelet deposition in the elbow regions (region $\mathrm{H}$ and region $\mathrm{K}$ ). The platelet deposition near the entrance region (region $\mathrm{G}$ ) is attributed to the nonideal inlet flow patterns (adapted from Ref. 12). 


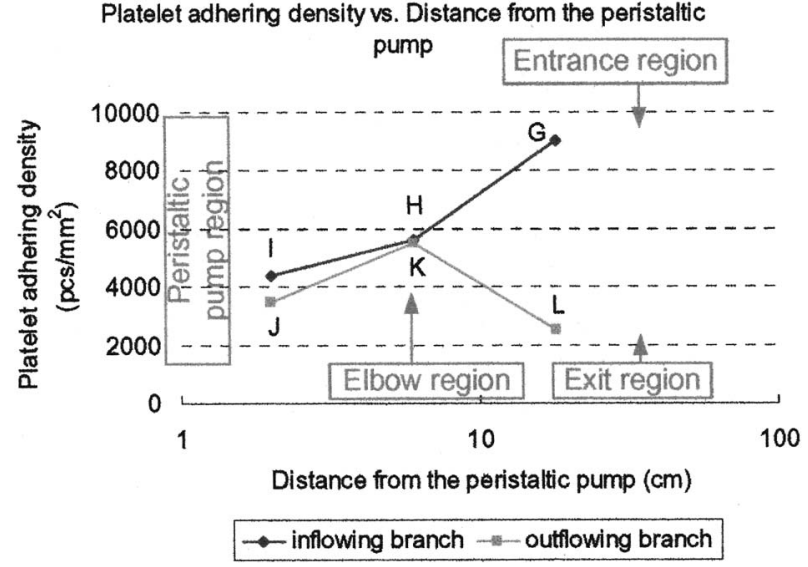

FIG. 4. The relation between the platelet adhering density (unit: pieces/ $\mathrm{mm}^{2}$ ) and the distance from the peristaltic pump (unit: $\mathrm{cm}$ ). The letters next to the points of the curves refer to the labeled sections shown in Fig. 2. The platelet adhesion shows a spatial decay as the blood flows away from the entrance region with the exception found at the outflowing elbow (regions $\mathrm{E}$ and $\mathrm{K})$.

We write the momentum and continuity equations as follows:

$$
\begin{aligned}
& \frac{\partial}{\partial t}\left(\rho u_{i}\right)+\frac{\partial}{\partial x_{j}}\left(\rho u_{i} u_{j}\right)=\frac{\partial p}{\partial x_{i}}+\frac{\partial \tau_{i j}}{\partial x_{j}}+\rho g_{i}+F_{i}, \\
& \frac{\partial \rho}{\partial t}+\frac{\partial}{\partial x_{i}}\left(\rho u_{i}\right)=0
\end{aligned}
$$

where $\rho$ is the fluid density, $u_{i}$ is the velocity in the direction $x_{i}, p$ is the pressure, $\rho g_{i}$ and $F_{i}$, are the gravitational and external body forces, respectively. $\tau_{i j}$ is the stress tensor, which is given in Eq. (5) below,

$$
\tau_{i j}=\left[\mu\left(\frac{\partial u_{i}}{\partial x_{j}}+\frac{\partial u_{j}}{\partial x_{i}}\right)\right],
$$

where $\mu$ is the blood viscosity, assuming that the blood is an incompressible Newtonian fluid.

We assume that there are no reacting agents that would cause an imbalance of forces throughout the domain of validity of the simulation. For simplicity, the tubing is assumed to be rigid so that the deformation of the internal tubing inside the peristaltic pump is ignored. Therefore, the pressure rise produced by the peristaltic pump is implicitly determined by the boundary condition at the entrance, where the constant inlet velocity is maintained. In addition, the bodyforce terms were neglected in the NS equation. Finally, no source terms were included in the continuity equation.

A commercial CFD package, FLUENT (Fluent Inc., Lebanon, $\mathrm{NH}$ ), was selected for the numerical simulation because of the robust and accurate models that are provided in its suite of simulation codes. A zero-pressure boundary condition was assumed at the exit of the loop into the blood reservoir. Also, a zero velocity (no-slip condition) was assumed at the walls. Because of the low Reynolds number $(\approx 100$ in the tubing), we assumed that turbulence effects can be neglected.

According to previous results, ${ }^{4}$ shear-induced platelet activation was found to be independent of the tubing surface-

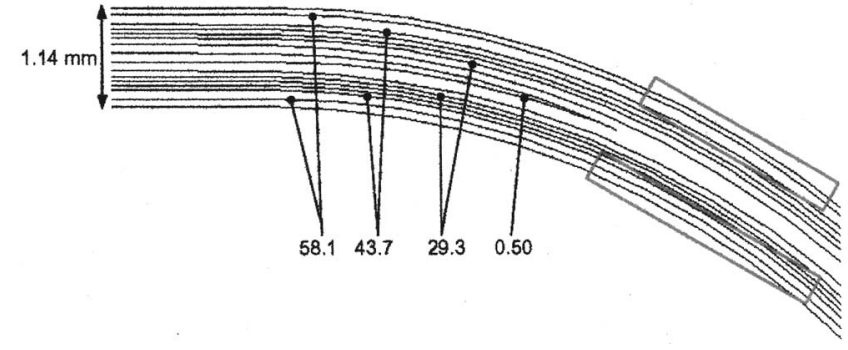

FIG. 5. Contours of constant shear rate (unit: 1/s) for the circulation loop system (at regions $\mathrm{E}$ and $\mathrm{K}$ ). The numbers refer to the values of shear rate on the contours as indicated. The strain rate reached a maximum value of $58.1 \mathrm{~s}^{-1}$ near the wall after about $1 \mathrm{~s}$ of operation. The rectangular regions are the locations where the platelets are likely to deposit.

to-volume ratio but dependent on the cumulative effects of the shear-rate variation and the blood exposure time. To quantify this, we specify an activation threshold index such that if the index is above this value, the platelets and/or the red blood cells activate. This is based on the experimental observations for platelet activation shown in Fig. 1 (Refs. 4 and 5) and is defined by numerically integrating $\int \dot{\gamma}(t) d t$ along a platelet trajectory. Since other studies ${ }^{5,7,8}$ suggest that flow separation and reattachment due to recirculation can also enhance platelet deposition, examination of the flow patterns and determination of their relationship to the activation level in the peristaltic recirculation system was also performed.

The activation level (stimulation) is the numerical integral of shear rate, and exposure time is given as ${ }^{4,5}$

$$
s=\int \dot{\gamma}(t) d t,
$$

where $s$ is the activation level and is dimensionless and $\dot{\gamma}(t)$ is the shear rate that a platelet experiences during an incremental exposure time interval $d t$. Since the microfluidic flow is assumed to be laminar, it is reasonable to assume that both the shear rate and the time the platelets are exposed to the shear field can be replaced by their spatially averaged values. For a constant shear flow, the activation level in the recirculation zone at which the procoagulant platelet factor 3 (FP3) is released is $s=1000{ }^{4,5}$ This threshold value for FP3 release can be used to determine whether platelets are activated before entering the transition regions (regions $\mathrm{C}$ and $\mathrm{D}$ ) shown in Fig. 2 where the tubing diameter changes. It is in these regions that secondary-flow patterns, such as recirculation or vortex shedding, can be created and are likely to further promote platelet deposition on the downstream tubing wall. ${ }^{5-7}$ The maximum shear rate was found to be $58.1 \mathrm{~s}^{-1}$ and was located near the wall in the elbow regions of the outflowing and inflowing tubes (shown in Fig. 2). Contours of constant shear rate are shown in Fig. 5. They are much higher than the values when blood flows through the larger-diameter tube inside the peristaltic pump, so the shear-rate contours in this tubing are not shown in this figure.

The values of shear rate near the wall of the tubing reached $29-58 \mathrm{~s}^{-1}$, which indicates that the platelets were likely to be activated within 17-34 s after entering the transition region $(s>1000)$ since no reverse flows (negative ve- 


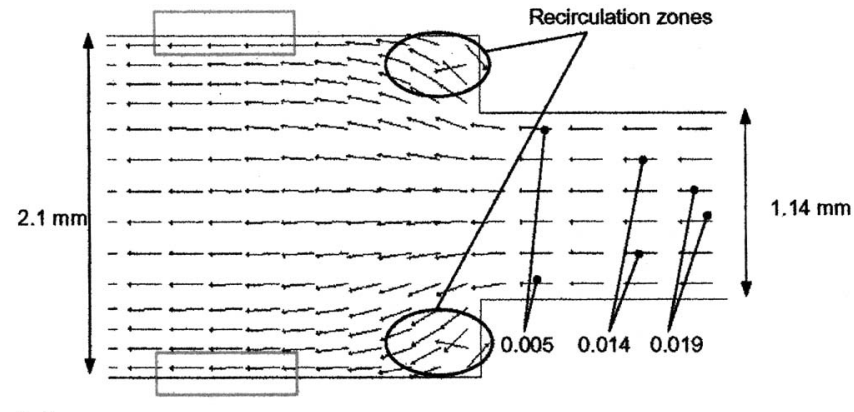

(a)

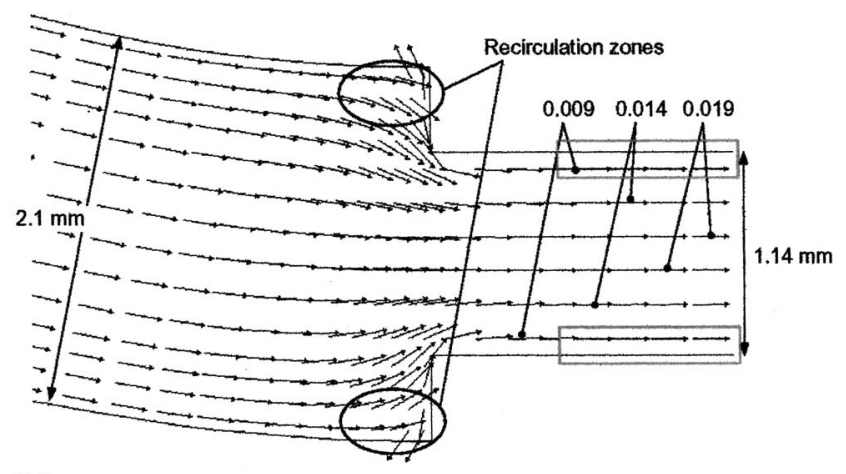

(b)

FIG. 6. Contours of constant wall shear stress (unit: Pascal) for the circulation loop system. Detailed contours of wall shear stress around the elbow region of the inflowing branch (regions $\mathrm{B}$ and $\mathrm{H}$ ), where the maximum value $(0.236 \mathrm{~Pa})$ was found close to the transition region, were selected in the figure. The numbers on the marked contours are values of the magnitude of wall shear stress on those contours. The number $0.18 \mathrm{~mm}$ indicates the boundary within where high wall shear stresses are present, which will result in strong interactions between platelets and the wall. The rectangular regions are the locations where the platelets are likely to deposit.

locities) were found at the section upstream of the transition region. This is within the time it takes for the blood to pass through the peristaltic pump, so the platelets are expected to remain activated even after leaving the pump. In our model circulation loop, the simulation shows that activation level $s$ was found to be greater than 1200 when the flow reached the transition region, which, from Fig. 1, predicts that platelets will be activated in the inflowing branch of the external tubing before entering the transition region.

The numerical results of wall-shear-stress distribution near the transition region, where the maximum value was found, are selected as shown in Fig. 6. Since the local wall shear stress could wash adhered platelets away from the wall if the stress is high, a nonuniform distribution of platelet deposition is expected at the locations downstream from the transition region.

For further investigation of the factors governing platelet deposition, the velocity field was examined because radial transport enhances collisions between the platelet aggregates, the coagulant agents, and the wall. ${ }^{2}$ Moreover, since the axial velocity of blood flow influences the growth of thrombi as they grow toward the center, this could cause elevated deposition of blood components. ${ }^{10}$ Contours of the axial and radial velocities as a function of distance along the tubing are shown in Fig. 7. The numerical results for the velocity field

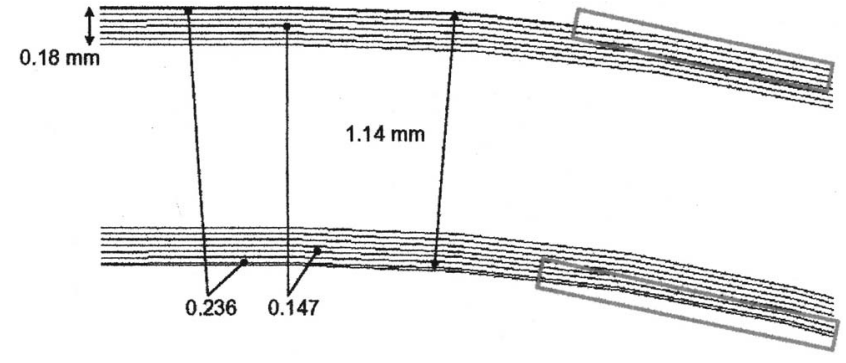

FIG. 7. Contours of constant axial velocity and radial velocity (unit: $\mathrm{m} / \mathrm{s}$ ) for the circulation loop system. (a) The marked contours show the values of velocity on those contours. The axial velocities (along region $\mathrm{D}$ to region $\mathrm{J}$ ) are larger at the elbow and transition regions. (b) The simulation results showed an increase of radial velocity, which increased the radial transport to the wall, in the sections between the elbow region and the transition region for both inflowing and outflowing branches. The rectangular regions are the locations where the platelets are likely to deposit.

show that axial velocities are larger when blood passes the elbow and transition regions, but the maximum values of the radial velocity are found only at the elbow regions (regions $\mathrm{H}$ and $\mathrm{K})$.

\section{DISCUSSION}

An earlier study ${ }^{16}$ that examined the redistribution of platelets in a blood-flow shear field found that red blood cells became more concentrated in the center of the flow as the shear rate increases, thus causing a concomitant decrease of platelet concentration in the center of the flow. In other words, a high blood-flow shear stress will expel platelets from the center toward the wall and promote their adherence. Since it would take more than $1 \mathrm{~min}$ for a platelet to finish the loop, the residence time of the platelets near the wall is long enough to enhance their adherence after their activation in the predicted 17-34 s. Furthermore, high shear stress will also enhance the activation of platelets near the tubing wall. ${ }^{17}$ Once the platelets are activated, they will likely adhere to each other and form aggregates, provided that the flow can create an environment for prolonged contacts between platelets with higher platelet collision frequency (which is a function of the volume of particles undergoing shear-induced collisions ${ }^{18}$ ) due to the increase in the hydrodynamic effective volume (HEV) of the platelets caused by changes in radii and/or shape produced by activation. The recirculation zones in our loop system are a good example for such environment. The HEV is defined as the volume occupied by the platelets with their effective radii before or after activation. ${ }^{2}$ The above studies outlined the influence of sheared flows on their blood components. We will characterize the flow parameters that govern the deposition of activated platelets in our loop system in detail in the following sections.

The simulation results for the velocity field in Fig. 8 show that recirculation zones are formed in the transition regions (regions $\mathrm{C}$ and $\mathrm{D}$ ). The combination of both high and low shears acting in the recirculation zones is conducive to the deposition of activated platelets, since in this location they will experience more interactions with the wall and thus will have increased chances to deposit on the wall. ${ }^{2}$ After 


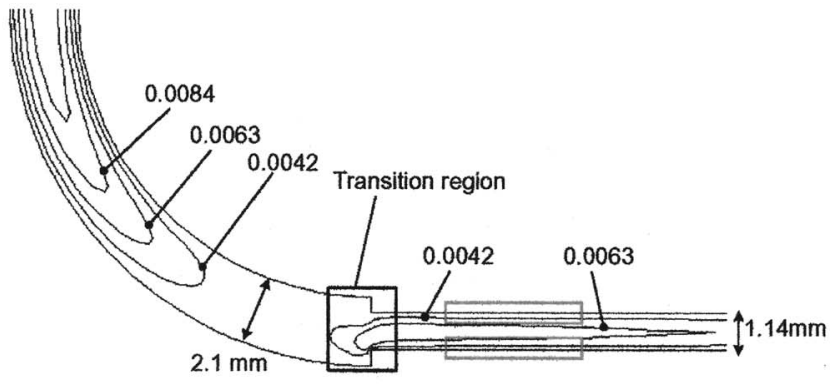

(a)

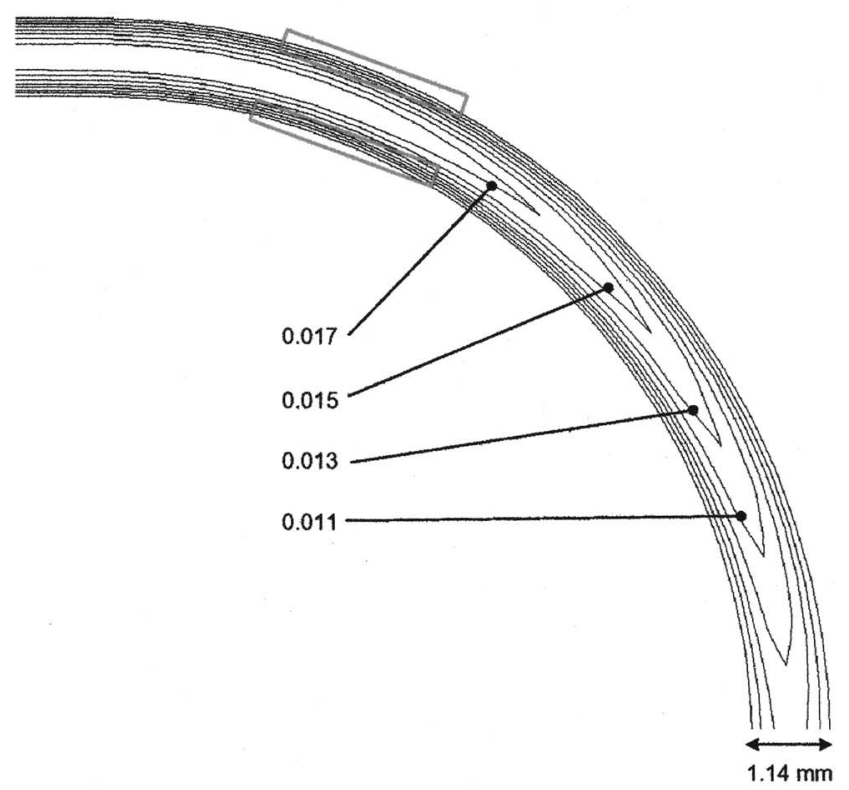

(b)

FIG. 8 . The velocity vector fields (unit: $\mathrm{m} / \mathrm{s}$ ) for the circulation loop system after blood flow in the tubing reached steady state. The numbers shown are values of the velocity magnitude on the marked contours. The detailed plots at (a) the transition region of the inflowing branch (region C) and (b) the transition region of the outflowing branch (region D) with the circular marks showing the recirculation zones. The recirculation zones formed around the transition region will bring the blood components towards the device wall. The rectangular regions are the locations where the platelets are likely to deposit.

entering and before leaving the larger tubing inside the peristaltic pump, where a slowly recirculating flow is expected to form due to the transition between the internal and external tubing, platelets activated in this region are more likely to promote thrombi after passing out of the transition region. As a result, aggregates of blood components were found deposited on the lumenal surface downstream from the transition region. ${ }^{19,20}$ In summary, we conclude that the transition region provides the environment for the development of platelet aggregates, and the nearest site downstream from the transition region, where the secondary flow reattaches to the mainstream, is where platelet aggregates are most likely to deposit.

The highest density of adhering platelets as shown in Fig. 4 was found at region $\mathrm{G}$, which is the downstream site just past the entrance region from the blood reservoir (region
A). The above result can be attributed to unsteady effects such as nonuniform flow and enhanced vorticity in the entrance region, where the velocity profile is still changing. The corresponding length of the affected region was estimated practically by several studies. ${ }^{21-24}$ For the flow condition in this model system, the length is approximately six times the diameter of the circular tube. This could increase platelet collision frequency at region $\mathrm{A}$, thus becoming part of the reason that a higher density of adhering platelets appears at its downstream region (region G). Furthermore, since the flow is always recirculating around the loop, it is possible that some of the aggregates that were not deposited can reenter the tubing from the reservoir. The above nonideal inlet flow patterns will then bring the platelets to the wall in the entrance region and its nearest downstream region (region G). Therefore, those reentered and still activated platelets continue to deposit on the wall close to the entrance.

Figure 4 also shows that the densities of adhering platelets decrease in moving from region $\mathrm{G}$ to region $\mathrm{H}$ (elbow region), and then to region I (upstream to the transition region). This occurs because the activated platelets entering the tubing from the reservoir were collected by previously deposited platelets on the lumenal surface. However, in the outflowing branch where the nonideal inlet effects were absent, the distribution of adhering platelets is determined mainly by the activation in the transition region and the deposition in the downstream elbow region. We hypothesize that the elevated density of platelet adhesion observed at the upstream elbow region (region $\mathrm{K}$ ) is attributed to the variation of axial and radial velocities in this region. ${ }^{10}$ The elevated axial velocity $(35 \%$ higher than the velocity at the entrance region) computed at the upstream transition region will thus likely produce a higher density of deposited platelets downstream of the transition region. ${ }^{10}$ Therefore, the density of platelet deposition at the elbow region (region $\mathrm{K}$ ) is expected to be larger than the one at its upstream transition region (region J). Another study also showed that significant near-wall excesses of platelets were found due to the migration of red blood cells toward the center of a laminar tube flow. ${ }^{25}$ This would cause a high concentration of platelets near the wall surface ${ }^{26}$ and help the transport of platelets toward the wall. Thus, the deposition of platelet aggregates on the wall surface around the elbow region is likely to increase with increased radial velocity, as shown in Fig. 7. In addition, since higher values of radial velocity exist in the elbow region, a higher density of platelet deposition is expected there due to higher radial transport toward the wall. This predicted result was verified by comparing the calculated flow patterns with the SEM images shown in Fig. 3.

According to the numerical results shown in Fig. 6, the distribution of the wall shear stress is highest near the wall of the transition region, so the density of platelet deposition is likely to be smaller on the lumenal surfaces downstream from the transition region since the adhered platelets will be washed away and deposited downstream from the transition region. It is likely that this is the reason that the density of platelet deposition is larger in region I than in region $\mathrm{J}$. 


\section{CONCLUSIONS}

Blood-carrying microtubing that has been subjected to surface-modification treatments on the lumenal surface can show qualitatively different results for platelet deposition. $^{15,27,28}$ Therefore, some modifications to the mechanism that blood components obey in interacting with the surface will be needed to account for these differences. However, as learned from this study, the aggregation of blood components was found to be closely dependent on the flow patterns, as influenced by the geometry of the experimental setup.

Previous studies ${ }^{2,5,7}$ indicated that separation and reattachment points, where secondary flows develop and diminish, respectively, enhanced the deposition of platelet aggregates. Other works also showed that the flow separation and recirculation zones promote the accumulation of coagulant agents near the wall, ${ }^{29}$ where a sharp concentration gradient across the flow streamlines that form the boundaries between the recirculation regions and the mainstream was found. From all the above statements, together with our numerical and experimental results, we can conclude that geometrical nonuniformities play a key role in the deposition of blood components since they lead to the variation of the velocity field and the formation of recirculation zones.

In order to predict and minimize unwanted aggregates, future devices should obtain the threshold for activation from a simulation and also include the effects introduced by pulsatile flows, flexible tubing, and surface interactions.

\section{ACKNOWLEDGMENT}

The authors wish to thank D. J. Klingenberg for several helpful suggestions.

${ }^{1}$ J. D. Hellums, Ann. Biomed. Eng. 22, 445 (1994).

${ }^{2}$ L. J. Wurzinger, P. Blasberg, and H. Schmid-Schöenbein, Biorheology 22 , 437 (1985)

${ }^{3}$ J. D. Hellums, D. M. Peterson, N. A. Stathopoulos, J. L. Moake, and T. R.
Giorgio, in Cerebral Ischemia and Hemorheology, edited by A. Hartmann and W. Kuschinsky (Springer-Verlag, New York, 1987).

${ }^{4}$ J. M. Ramstack, L. Zuckerman, and L. F. Mockros, J. Biomech. 12, 113 (1979).

${ }^{5}$ D. Bluestein, L. Niu, T. Schoephoerster, and M. K. Dewanjee, Ann. Biomed. Eng. 25, 344 (1997).

${ }^{6}$ R. L. Whitmore, Rheology of the Circulation (Pergamon, Oxford, 1968). ${ }^{7}$ D. Bluestein, G. Gutierrez, M. Londono, and T. Schoephoerster, Ann. Biomed. Eng. 27, 763 (1999).

${ }^{8}$ D. Bluestein, E. Rambod, and M. Gharib, J. Biomech. Eng. 122, 125 (2000).

${ }^{9}$ S. Raz, S. Einav, Y. Alemu, and D. Bluestein, [Engineering in Medicine and Biology, 2002. 24th Annual Conference and the Annual Fall Meeting of the Biomedical Engineering Society] EMBS/BMES Conference, 2002. Proceedings of the Second Joint (IEEE, Piscataway, NJ, 2002), Vol. 2, pp. 901-902.

${ }^{10}$ J. Strony, A. Beaudoin, D. Brands, and B. Adelman, Am. J. Physiol. 265, H1787 (1993).

${ }^{11}$ S. Sukavaneshvar, G. M. Rosa, and K. A. Solen, Ann. Biomed. Eng. 28, $182(2000)$

${ }^{12}$ S. I. Simon and H. L. Goldsmith, Ann. Biomed. Eng. 30, 315 (2002).

${ }^{13}$ H. L. Goldsmith, T. A. Quinn, G. Drury, C. Spanos, F. A. Mclntosh, and S. I. Simon, Biophys. J. 81, 2020 (2001).

${ }^{14}$ P. Tandon and S. L. Diamond, Biophys. J. 75, 3163 (1998).

${ }^{15}$ J. L. Lauer et al., J. Appl. Phys. 96, 4539 (2004).

${ }^{16}$ P. A. M. M. Aarts, S. A. T. van den Broek, G. W. Prins, G. D. C. Kuiken, J. J. Sixma, and R. M. Heethaar, Arteriosclerosis (Dallas) 8, 819 (1988).

${ }^{17}$ S. Sukavaneshvar, G. M. Rosa, and K. A. Solen, Ann. Biomed. Eng. 28, 182 (2000).

${ }^{18}$ P. Tandon and S. L. Diamond, Biophys. J. 73, 2819 (1997).

${ }^{19} \mathrm{H}$. Schmid-Schöenbein et al., Biorheology 18, 415 (1981).

${ }^{20}$ R. T. Schoephoerster, F. Oynes, G. Nunez, M. Kapadvanjwala, and M. K. Dewanjee, Arterioscler. Thromb. 13, 1806 (1993).

${ }^{21}$ H. L. Langhaar, J. Appl. Mech. 9, A55 (1942).

${ }^{22}$ B. Atkinson, M. P. Brocklebank, C. C. H. Card, and J. M. Smith, AIChE J. 15, 548 (1969).

${ }^{23}$ M. Friedmann, J. Gillis, and N. Liron, Appl. Sci. Res. 19, 426 (1968)

${ }^{24}$ R. Y. Chen, J. Fluids Eng. 95, 153 (1973).

${ }^{25}$ S. K. Wang and N. H. C. Hwang, Biorheology 29, 353 (1992).

${ }^{26}$ D. Basmadjian, Ann. Biomed. Eng. 18, 685 (1990).

${ }^{27}$ A. S. Kantak, B. K. Gale, T. Lvov, and S. A. Jones, Biomed. Microdevices 5, 207 (2003).

${ }^{28}$ T. N. Zaidi, L. V. Mclntire, D. H. Farrel, and P. Thiagarjan, Blood 88, 2967 (1996).

${ }^{29}$ E. M. Lutostansky, G. Karner, G. Rappitsch, D. N. Ku, and K. Perktold, J. Biomech. Eng. 125, 189 (2003). 